\title{
Ação do extrato de própolis na Leishmaniose
}

\section{Propolis extract action in Leishmaniasis}

\author{
Julie Massayo Maeda Oda ${ }^{1}$; Thiago Cezar Fujita²; Amanda de Fáveri Pitz²; Marlusa \\ Karlen Amarante ${ }^{2}$; Ionice Felipe ${ }^{3}$; Halha Ostrensky Saridakis ${ }^{4}$; José Mauricio \\ Sforcin $^{5}$; Maria Angelica Ehara Watanabe ${ }^{3}$; Ivete Conchon Costa ${ }^{3}$
}

\begin{abstract}
Resumo
A leishmaniose causa mortalidade e morbidade em mais de 80 países e caracteriza-se como uma doença parasitária com diversas manifestações, por isso constitui um sério problema de saúde pública. No Novo Mundo ocorre a Leishmaniose Visceral (LV) e Leishmaniose Tegumentar Americana (LTA). A LTA é considerada uma enfermidade polimórfica, espectral da pele e das mucosas, agrupada em diferentes formas clínicas: a leishmaniose cutânea, a cutaneomucosa e a cutânea difusa. A Organização Mundial da Saúde recomenda os antimoniais pentavalentes como drogas de primeira escolha no tratamento da leishmaniose. Entretanto, devido aos efeitos indesejados dessas drogas, tem sido proposto o estudo para o desenvolvimento de novos fármacos para seu tratamento. Assim, tem-se investigado o uso da própolis, visto que está relacionada a muitas atividades biológicas, como antibacteriana, antifúngica, antiulcerativa, antiviral, antiprotozoária, antiinflamatória, hepatoprotetora, antioxidante, antitumoral, entre outras. Estudos recentes mostraram que a própolis verde ativa macrófagos e estimula os linfócitos $\mathrm{B}$ a produzirem anticorpos. A própolis tem demonstrado potencial atividade leishmanicida, por diminuir o diâmetro das lesões, causar alterações morfológicas nas formas promastigotas ou ainda por melhorar a resposta imunológica frente às Leishmanias, ativando macrófagos.Portanto, o objetivo deste trabalho foi relatar as principais atividades leishmanicidas da própolis, por meio de pesquisa bibliográfica eletrônica no PubMed e Scielo durante o ano de 2009
\end{abstract}

Palavras-chave: Leishmaniose. Própolis. Ação antiprotozoária.

\begin{abstract}
Leishmaniasis is a public health problem causing morbidity and mortality in over 80 countries and features a parasitic disease with several manifestations. In the New World occur Visceral Leishmaniasis (VL) and American Cutaneous Leishmaniasis (ACL). The ACL is considered a disease polymorphic of skin and mucous membranes, grouped in different clinical forms: cutaneous leishmaniasis in the skin and mucosa and diffuse cutaneous, and the evolution of this disease is closely related to the mechanisms of escape of the protozoan Leishmania from the immune response, allowing the development of the disease and consequently the onset of clinical manifestations known. The World Health Organization recommends the pentavalent antimony as the first choice drugs. However, they usually give unsatisfactory results, requiring the development of new and affordable drugs for its treatment. It has been investigated that use of propolis it is related to exhibit several biological activities such as antibacterial, antifungal,
\end{abstract}

\footnotetext{
1 Bacharel em Biomedicina, Universidade Estadual de Londrina; Mestre em Patologia Experimental, Universidade Estadual de Londrina; Doutoranda do Programa de Pós-Graduação em Patologia Experimental, Universidade Estadual de Londrina. Londrina - PR. E-mail: julie massayo@hotmail.com

2 Mestrandos do Programa de Pós-Graduação em Patologia Experimental, Universidade Estadual de Londrina. Londrina - PR.

3 Docente lotado no Departamento de Ciências Patológicas, Centro de Ciências Biológicas, Universidade Estadual de Londrina. E-mail: conchon@uel.br

4 Docente lotado no Departamento de Microbiologia, Centro de Ciências Biológicas, Universidade Estadual de Londrina.

5 Docente lotado no Departamento de Patologia, Unesp/Botucatu.
} 
anti-ulcer agents, antiviral, anti-protozoan, anti-inflammatory, hepatoprotective, antioxidant, antitumor, among others. Recent studies have shown that green propolis active macrophages and stimulates lymphocytes B to produce antibodies. It was also reported that propolis shows a potential antileishmanial activity by reducing the diameter of the lesions, avoiding complications of the lesions by bacteria or by improving the immune response against the Leishmania through macrophages activation. The aim of this work was related the main activities leishmanicida using propolis, by searched the electronic literature PubMed and Scielo during the year 2009.

Key words: Leishmaniasis. Propolis. Anti-protozoan activity

\section{Introdução}

Leishmaniose é um grupo de doenças tropicais causadas por um amplo número de espécies de protozoários parasitas pertencentes ao gênero Leishmania (KAYSER; KIDERLEN; CROFT, 2003). De acordo com a Organização Mundial da Saúde (WORLD HEALTH ORGANIZATION, 2002), a leishmaniose está entre as seis mais importantes doenças tropicais, afetando cerca de 12 milhões de pessoas em 88 países (BAILEY; LOCKWOOD, 2007), com aproximadamente 350 milhões de pessoas em risco e 2.357 .000 novos casos por ano. Espécies de Leishmania do Velho Mundo causam patologias no sul da Europa e Ásia, enquanto as espécies do Novo Mundo são encontradas na América do Sul, Central e sul dos Estados Unidos. No Brasil, há aproximadamente 26.000 casos de leishmaniose registrados por ano, sendo que a maior parte da população vive em áreas onde a leishmaniose é endêmica, como a região Norte do Brasil (NETO et al., 2004).

São conhecidas várias espécies que causam infecção em humanos. Como as diferentes espécies são indistinguíveis morfologicamente, vários critérios têm sido adotados para sua identificação, utilizando-se características extrínsecas (aspectos clínicos, distribuição geográfica, comportamento em cultura e em animais de laboratório ou vetores) e intrínsecas (critérios imunológicos, bioquímicos ou moleculares) (LUMSDEN, 1974; GRIMALDI; TESH; MCMAHON-PRATT, 1989; RIOUX et al., 1990).

A leishmaniose no Novo Mundo ocorre na forma de Leishmaniose Visceral (LV) causada pela L. chagasi, e Leishmaniose Tegumentar
Americana (LTA) com o envolvimento de várias espécies de Leishmania. No Brasil, circulam as espécies: Leishmania (Leishmania) amazonensis, Leishmania (Viannia) guyanensis, Leishmania (V.) braziliensis, Leishmania (V.) shawi, Leishmania (V.) naiffi e Leishmania (V.) lainsoni (GENARO, 2005; CASTRO et al., 2007). No Estado do Paraná, Silveira et al. (1999) ressaltaram a presença de Leishmania (Viannia) braziliensis e Leishmania (Leishmania) amazonensis.

O gênero apresenta morfologia variável quanto ao hospedeiro que infecta: a) encontrado nas formas flageladas, promastigotas e paramastigotas, no trato digestivo dos hospedeiros invertebrados $\mathrm{e}$ b) amastigotas, parasita intracelular obrigatório do sistema fagocítico mononuclear do hospedeiro vertebrado (GENARO, 2005). Essas formas são capazes de se multiplicar, lisar a célula hospedeira e infectar novos macrófagos (AYRES; MARCUCCI; GIORGIO, 2007).

A leishmaniose tegumentar americana é considerada uma enfermidade polimórfica, espectral da pele e das mucosas, agrupadas nas diferentes formas clínicas: a leishmaniose cutânea, a cutaneomucosa e a cutânea difusa (AMIN; MANISALI, 2000; ANTONOVICH, 2004).

Segundo Neves (2000), a forma cutânea é caracterizada pela formação de úlceras únicas ou múltiplas na derme com a epiderme ulcerada, e resulta em úlceras leishmanióticas típicas ou, então, evolui para formas vegetantes verrucosas. A forma cutaneomucosa apresenta lesão inicial primária, lesões destrutivas secundárias que envolvem mucosas e cartilagens, e se trata, pois, de um 
processo lento, de curso crônico. As regiões mais afetadas são o nariz, a faringe, a boca e a laringe, em muitos casos ocorre a destruição de toda a estrutura cartilaginosa do nariz. O processo ulcerativo pode atingir lábios e propagar-se pela face (BARRAL et al., 1993; MODABBER, 1993). A forma cutânea difusa caracteriza-se pela formação de lesões difusas não ulceradas envolvendo amplas áreas da pele, particularmente extremidades e outras partes expostas onde numerosas erupções papulares ou nodulares não ulceradas são vistas. A doença caracteriza-se por curso crônico e progressivo durante toda a vida do paciente (NEVES, 2000).

Osquadros clínicos apresentados na leishmaniose estão relacionados tanto à espécie de Leishmania envolvida como também à resposta imune desenvolvida pelo hospedeiro (ZIMMERMANN et al., 2009). As formas amastigotas das leishmanias, encontradas nos hospedeiros vertebrados, são intracelulares obrigatórias de células do sistema fagocítico mononuclear, e induzem uma complexa interação com a resposta imune do hospedeiro (HALL; JOINER, 1991).

Estudos com infecção experimental em camundongos suscetíveis e resistentes infectados com Leishmania, principalmente L. major, têm contribuído grandemente para o entendimento do papel da resposta imune mediado por células na leishmaniose (TRIPATHI; SINGH; NAIK, 2007).

Segundo De Almeida, Cardoso e Barral-Neto (2003), o modelo murino é o mais frequentemente empregado no estudo de infecções com Leishmania, mas apresenta uma série de diferenças importantes em relação à doença humana, o que limita a possibilidade de extrapolação para a infecção humana. Contudo, os autores salientam que a aplicação destes modelos ainda é essencial para estudos da leishmaniose.

Scott et al. (1988) demonstraram que, em infecções experimentais por $L$. major em camundongos geneticamente resistentes, os animais desenvolviam uma resposta com predomínio do padrão Th1, caracterizado pela secreção de IFN- $\gamma$, enquanto que em camundongos suscetíveis à resposta dominante era o padrão Th2, caracterizado por secreção de citocinas IL-4, IL-5 e IL-13.

Wang et al. (1994) demonstraram experimentalmente a função de IFN- $\gamma$ no controle da infecção por $L$ major utilizando camundongos resistentes C57BL/6 modificados geneticamente $(\mathrm{IFN}-\gamma-/-)$ e submetidos à infecção com $L$. major. Esses animais deficientes em IFN- $\gamma$ foram incapazes de restringir o crescimento do parasita e sofreram infecção letal. A secreção de IFN- $\gamma$ leva à morte intracelular das formas amastigotas através da ativação de macrófagos, associado à indução de óxido nítrico (NO) sintase, e morte mediada por NO (LIEW et al., 1990). Outros estudos têm demonstrado a função crítica de outras citocinas como IL-12, IL-10 e TNF- $\alpha$ na suscetibilidade ou resistência à leishmaniose tegumentar (MATTNER et al., 1996; REED; SCOTT, 1993; BELKAID et al., 2002; PADIGEL; ALEXANDER; FARRELL, 2003; PADIGEL; FARRELL, 2003; PADIGEL et al., 2003; VON STEBUT et al., 1998, SOLBACH; LASKAY, 2000; NOBEN-TRAUTH; KROPF; MÜLLER, 1996, RITTER et al., 2004a, 2004b, ROBERTS, 2006).

Embora seja montada uma resposta imune frente a esse parasita, a evolução da doença também está relacionada aos mecanismos de escape do protozoário Leishmania frente à resposta imune desenvolvida contra ele. Entre esses mecanismos, mencionam-se: as moléculas de superfície, a inibição das funções dos macrófagos, a indução e a secreção de moléculas imunossupressoras e a alteração da sinalização celular do hospedeiro (CASTELLANO et al., 1999; REIS et al., 2006). Isso permite ao parasita evadir da resposta imune inata e o torna capaz de dividir-se nos macrófagos infectados, possibilitando a disseminação da doença no hospedeiro.

A quimioterapia da leishmaniose tem sido baseada no uso de drogas antimoniais pentavalentes, 
como o gluconato de antimônio(V) sódico (Pentostan ${ }^{\circledR}$ ) e N-metilglucamina (Glucantime ${ }^{\circledR}$ ), e o Ministério da Saúde recomenda estes antimoniais pentavalentes como drogas de primeira escolha (MINISTÉRIO DA SAÚDE, 2000). Entretanto, pode haver resultados insatisfatórios, devido à resistência à droga, falta de eficácia, toxicidade, dificuldade de administração, tratamento prolongado e o alto custo. Outros medicamentos, tais como pentamidina e anfotericina $\mathrm{B}$, têm sido empregados como drogas alternativas. Entretanto, estas drogas necessitam de administração parenteral a longo prazo e exibem efeitos secundários graves (KAYSER; KIDERLEN; CROFT, 2003). A ação sinérgica entre drogas e a resposta imune do hospedeiro têm sido amplamente investigadas através do uso de drogas e imunoestimulantes (EL-ON; HAMBURGER, 1987). Pesquisas com a leishmaniose no Brasil se explicam não apenas por sua alta incidência e ampla distribuição, mas também pela possibilidade de a doença assumir formas graves e letais. Isso justifica a pesquisa de novas drogas terapêuticas leishmanicidas e para o entendimento da relação deste protozoário com o hospedeiro humano.

\section{Própolis}

A própolis é um produto natural, elaborado pelas abelhas (Apis mellifera) a partir de material coletado em botões, gemas e córtex vegetais, para ser utilizada como uma barreira protetora na colméia. Apresenta um conjunto complexo de substâncias constituído basicamente por resinas e bálsamos $(55 \%)$, cera $(35 \%)$, óleos vegetais $(10 \%)$ e pólen (5\%), possuindo grande variedade de compostos fenólicos, principalmente flavonóides, atribuído aos diferentes tipos de plantas utilizadas pelas abelhas. Além dos flavonóides, podem ser encontrados em sua composição ácidos aromáticos, ésteres, aldeídos, terpenos, aminoácidos, polissacarídeos, hidrocarbonetos, álcoois, hidroxibenzenos, dentre outros (MARCUCCI, 1995; BANKOVA;
CASTRO; MARCUCCI, 2000). Contudo seus constituintes químicos biologicamente ativos são altamente variados dependendo da flora, área geográfica e estação da coleta (KUJUMGIEV et al., 1999; BANKOVA; CASTRO; MARCUCCI, 2000).

A própolis do Sudeste brasileiro, conhecida como própolis verde devido à sua cor, é extensivamente utilizada em alimentos e bebidas, especialmente no Brasil e no Japão, com o objetivo de melhorar a saúde e prevenir muitas doenças (MARCUCCI, 1995; MARCUCCI; DE CAMARGO; LOPES, 1996; CAMPOS et al., 1998, BANKOVA; CASTRO; MARCUCCI, 2000; MISSIMA et al., 2007). No Brasil, a planta mais importante como fonte de própolis verde (BGP) é a Baccharis dracunculifolia D. C. (Asteraceae) (KUMAZAWA et al., 2003).

Vários autores têm relatado as atividades biológicas da própolis, como antibacteriana, antifúngica e antiviral (KUJUMGIEV et al., 1999; SFORCIN et al., 2000, SFORCIN; ORSI; BAKOVA, 2005, MURAD et al., 2002, LEITÃO et al., 2004; ORSI; BANKO; SFORCIN, 2005, SALOMÃO et al., 2008), antiulcerativa (BARROS et al., 2007), anti-inflamatória (REIS et al., 2000), antimutagênica (SCHELLER et al., 1989; TAVARES et al., 2006), antioxidante (SIMÕES et al., 2004), tripanomicida (MARCUCCI et al., 2001; DA SILVA FILHO et al., 2004a, 2004b), imunoestimulatória (TATEFUJI et al., 1996; SFORCIN; ORSI; BANKOVA, 2005, MISSIMA et al., 2007).

Em relação à leishmaniose, recentemente alguns pesquisadores têm demonstrado o efeito de diferentes extratos da própolis tanto in vitro com in vivo. Machado, Leon e De Castro (2007) testaram diferentes extratos etanólicos de própolis, um brasileiro (Et-Bra) e um búlgaro (Et-Bgl) e um extrato com acetona da Bulgária (Ket-Bgl). Essas amostras foram testadas em experimentos in vitro, nas concentrações $0,5 \mu \mathrm{g} / \mathrm{mL}$ a $500 \mu \mathrm{g} / \mathrm{mL}$, 
contra formas promastigotas de quatro espécies de Leishmania, sendo três do Novo Mundo ( $L$. amazonensis, L. braziliensis, L. chagasi) e uma do Velho Mundo (L. major). Os extratos búlgaros Et-Bgl apresentaram atividade decrescente contra os parasitas: L. major $>$ L. amazonensis $>L$. chagasi $>$ L. braziliensis. O extrato brasileiro apresentou atividade similar frente às espécies $L$. major, L. braziliensis e L. chagasi, enquanto que L. amazonensis foi a espécie menos suscetível.. Salomão et al. (2004) haviam associado alta atividade microbicida do Et-Blg devido a grandes concentrações de flavonóides. Marcucci e Bankova (1999) haviam previamente observado diferente composição química entre as amostras de própolis do Brasil e da Bulgária: o extrato da Bulgária apresentava predominância de flavonóides, enquanto o extrato brasileiro apresentava apenas $1,1 \%$. Contudo, como ambos os extratos apresentaram atividade similar contra $L$. chagasi e o Et-Bra foi mais ativo contra L. braziliensis, os autores sugeriram que outros componentes, além dos flavonóides, estariam envolvidos na atividade leishmanicida.

Duran et al. (2008) analisaram a atividade in vitro da própolis nas concentrações de 25, 50, 100 , 250,500 e $750 \mu \mathrm{g} / \mathrm{mL}$ contra promastigotas de 5 isolados clínicos de Leishmania tropica. Os autores avaliaram o crescimento dos parasitas, viabilidade celular (mobilidade dos parasitas) e morfologia celular. Quanto à morfologia, verificaram que não houve efeito patológico nas concentrações de 25 , 50 e $100 \mu \mathrm{g} / \mathrm{mL}$, e observaram aumento no número de parasitas em todos os tempos analisados. Entretanto, as concentrações de 250, 500 e 750 $\mu \mathrm{g} / \mathrm{mL}$ apresentaram efeito dose-dependente em todos os tempos analisados e foi observada grande diminuição da viabilidade celular das promastigotas e diversas alterações morfológicas como granulações e perda de flagelo. Os autores acreditam que o efeito observado deste extrato etanólico pode ser devido aos terpenoides ou hidrocarbonos e ácidos graxos, uma vez que esse extrato, segundo Sahinler e Kaftnanoglu (2005), apresenta altas concentrações desses componentes.

Em trabalho realizado por Pontin et al. (2008), os autores avaliaram o efeito leishmanicida in vitro do extrato hidroalcoólico de própolis verde contra Leishmania (Viannia) braziliensis. As formas promastigota e amastigotas intracelulares foram tratadas com diferentes concentrações do extrato $(1,10,30,50,100,250,500$ e 750 $\mu \mathrm{g} / \mathrm{mL}$ para promastigotas e 10,100 e $250 \mu \mathrm{g} /$ $\mathrm{mL}$ para amastigotas intracelulares). Após a incubação, verificou-se $79,3 \%$ de lise das formas promastigotas na concentração de 500 $\mu \mathrm{g} / \mathrm{mL}$, enquanto a anfotericina B lisou $68,3 \%$ dos parasitas nesta mesma concentração. Para as formas amastigotas intracelulares, o extrato hidroalcoólico de própolis verde foi inativo. Os autores avaliaram, ainda, o efeito leishmanicida do extrato da própolis verde brasileiro in vivo sobre a L. braziliensis. Camundongos BALB/c receberam injeção subcutânea na base da calda com $2 \times 10^{6}$ formas promastigotas, após o aparecimento da lesão os camundongos foram divididos em três grupos: o primeiro recebeu apenas tratamento oral, outro tratamento tópico e o outro recebeu os dois tratamentos concomitantes, todos na concentração de $1,5 \mathrm{mg} / \mathrm{kg} / \mathrm{dia}$, e o diâmetro da lesão foi medido mensalmente. Como controle positivo, utilizaram glucantime intraperitoneal na concentração de 20 $\mathrm{mg} /$ dia e o controle negativo recebeu $\mathrm{NaCl} 0,9 \%$. $\mathrm{O}$ tratamento oral com extrato de própolis em camundongos infectados com Leishmania acarretou redução significativa $(78,6 \%)$ no diâmetro das lesões, e o tratamento tópico com o mesmo reduziu em $84,3 \%$ e que o tratamento concomitante, oral e tópico do extrato, causou uma redução considerável (90\%) no diâmetro das lesões. Já o tratamento com glucantime reduziu $57,7 \%$ do tamanho das lesões, mostrando o efeito leishmanicida deste extrato de própolis tanto in vitro como in vivo.

Ayres, Marcucci e Giorgio (2007) investigaram o efeito do extrato de propolis coletado no Estado de Alagoas (BRV - própolis vermelha) sobre formas 
promastigotas e formas amastigotas obtidas de lesão da espécie L. (L.) amazonensis. Esse extrato contém altas concentração de componentes prenilados e benzofenonas (MARCUCCI; BANKOVA, 1999; MARCUCCI et al., 2001). As formas promastigotas e amastigotas, foram incubadas com $25 \mu \mathrm{g} / \mathrm{mL}$ de própolis e o número de parasitas e a morfologia foram avaliados. Até o período de 96 horas, os autores não observaram alteração na proliferação tanto das formas promastigotas como das formas amastigotas, portanto este extrato não se mostrou eficiente diretamente contra tais formas. Contudo, quando os autores utilizaram este extrato e dois outros provenientes do estado do Paraná, BRG e BRPG (própolis verde) e um de Minas Gerais BRP-1 (própolis verde) em culturas de macrófagos murinos (camundongos BALB-c) infectados com L. amazonensis, houve diminuição tanto na porcentagem de macrófagos infectados como no número de amastigotas no interior dos macrófagos.

Macrófagos infectados e incubados com os extratos BRG e BRPG. na concentração de $25 \mu \mathrm{g} /$ $\mathrm{mL}$ por 72 horas apresentaram redução no número de macrófagos infectados e número de parasitas intracelulares.

Em relação ao extrato BRV e BRP-1, os autores utilizaram as concentrações de 3, 6, 12,5, 25, 50 e $100 \mu \mathrm{g} / \mathrm{mL}$ durante períodos de 24, 48 e 72 horas. Verificou-se a capacidade de reduzir significativamente a infecção de macrófagos nas concentrações de $6 \mu \mathrm{g} / \mathrm{mL}$ a $100 \mu \mathrm{g} / \mathrm{mL}$. Os autores observaram que concentrações de $6 \mu \mathrm{g} / \mathrm{mL}$ do extrato BRV reduziram em $84,5 \%$ o nível de infecção e em concentrações maiores nenhum macrófago foi encontrado infectado. Esses resultados permitiram sugerir que constituintes da própolis intensificaram os mecanismos microbicidas dos macrófagos, levando à morte da L. amazonensis. Contudo, neste mesmo experimento, os autores observaram que concentrações superiores a $25 \mu \mathrm{g} / \mathrm{mL}$ de BRG e BRPG foram tóxicas para os macrófagos.

Vários outros autores investigaram a capacidade de diversos extratos de própolis em causar dano ao DNA. Pereira e Alves (2008), utilizando diferentes doses do extrato de própolis verde e os testes de cometa e micronúcleo em células de sangue periférico de camundongos, demonstraram um aumento no dano ao DNA dessas células. Estudos de mutagenicidade e citotoxicidade in vitro apresentaram resultados positivos com extrato da própolis verde (TAVARES et al., 2006; LIMA, 2007; BANSKOTA et al., 1998; BANSKOTA et al., 2000), e aconselharam o uso moderado desse extrato, salientando a necessidade do desenvolvimento de outros testes mutagênicos, para, finalmente, estabelecer as dosagens que possam representar risco a saúde dos seres humanos.

Os mecanismos pelos quais o extrato de própolis exerce seu efeito leishmanicida in vivo não estão esclarecidos. Tem sido sugerido que o efeito leishmanicida do própolis pode ser devido, pelo menos em parte, à ativação de macrófagos. Sabese que os macrófagos estão envolvidos em muitas funções, como fagocitose, liberação de enzimas, geração de radicais livres e mediadores de processos inflamatórios.

Orsi et al. (2000), investigaram o efeito da própolis verde brasileiro, solução de própolis hidroalcoólica (PHS) na ativação de macrófagos peritoneais de camundongos BALB/c com ensaios in vitro e in vivo. Foram testadas as concentrações de 2.5, 5, 10, 20, 50 e $100 \mu \mathrm{g} / \mathrm{mL}$ de PHS no ensaio in vitro, os autores observaram que a própolis não mostrou aumento significativo na produção de $\mathrm{H}_{2} \mathrm{O}_{2}$ e óxido nítrico. Nos testes in vivo foram utilizadas as concentrações de 250, 500, 1000, 3000 e $6000 \mu \mathrm{g} / \mathrm{mL}$ de própolis, observando que nos camundongos tratados com própolis houve indução na produção de óxido nitrico de maneira dose dependente. A produção de NO foi estimulada nos macrófagos quando os animais eram tratados com as concentrações de 250, 500 e $1000 \mu \mathrm{g} / \mathrm{mL}$ de própolis e inibida nas concentrações de 3000 e $6000 \mu \mathrm{g} / \mathrm{mL}$ de BGP, e isso sugere que a própolis apresenta um importante papel na imunidade não específica, por ativar macrófagos. 
Além da atividade imunomoduladora em macrófagos, Sforcin, Orsi e Bankova (2005) reportaram os resultados de seu estudo sobre a ação da própolis brasileira e búlgara na produção de anticorpos em ratos imunizados com albumina sérica bovina (BSA), verificando aumento na produção de anticorpos 15 dias após a imunização. Os autores não observaram diferença estatística na produção de anticorpos nos dois extratos testados, independentemente da origem geográfica e da estação de coleta, demonstrando também efeito imunomodulador da própolis na produção de anticorpos.

\section{Conclusão}

A própolis tem mostrado efeito protetor contra as várias espécies de Leishmania estudadas, seja por atuar diretamente sobre as formas promastigotas e/ ou amastigotas, por agir na produção de substâncias microbicidas pelos macrófagos ou ainda por diminuir o diâmetro das lesões em experimentos in vivo como pode ser observado no esquema da Figura 1.

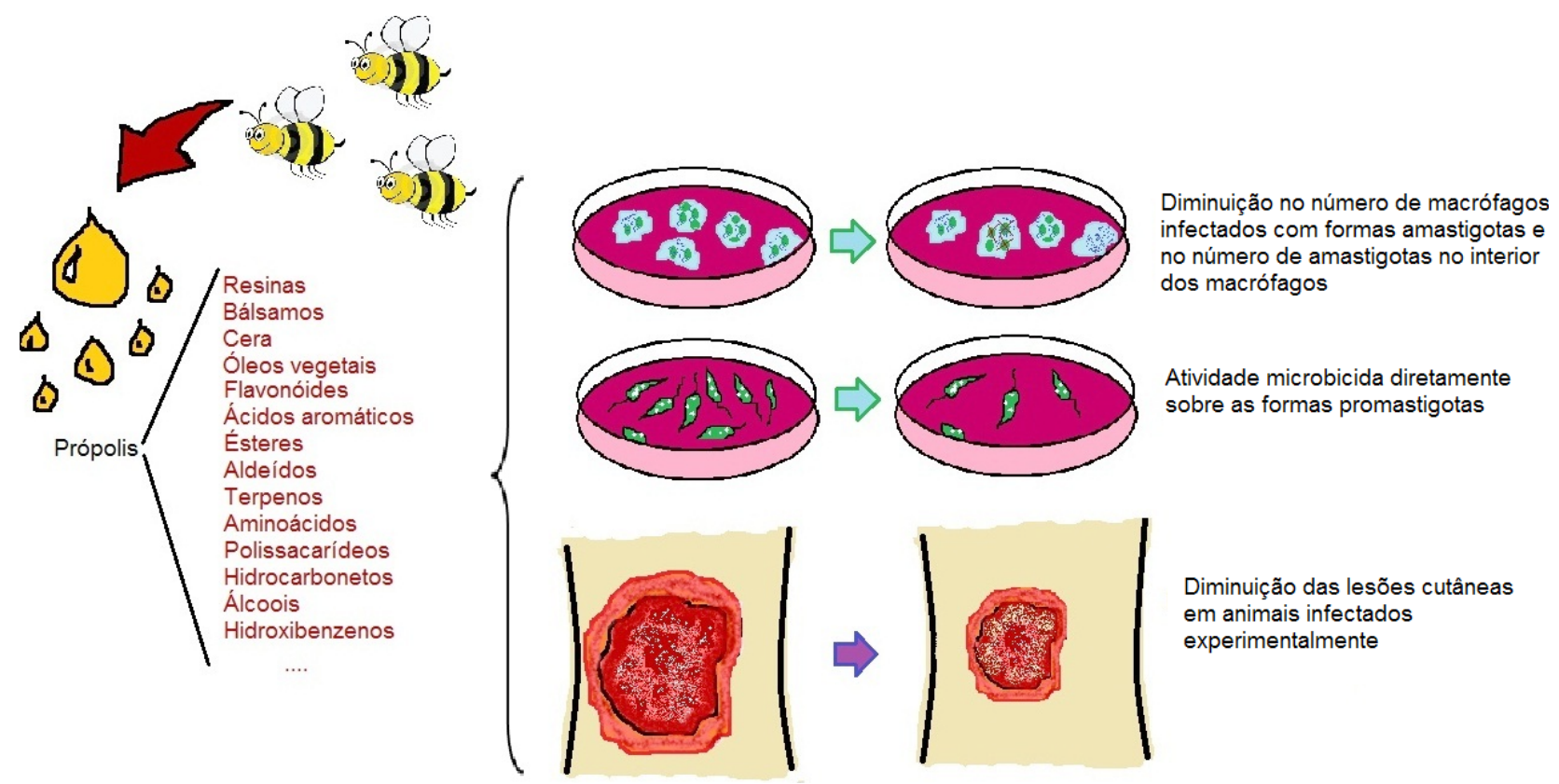

Figura 1. Esquema de possíveis mecanismos de ação da própolis in vitro e in vivo na leishmaniose.

\section{Referências}

AMIN, M.; MANISALI, M. Cutaneous leishmaniasis affecting the face: report of a case. Journal of Oral and Maxillofacial Surgery, Philadelphia, v. 58, n. 9, p. 10661069, 2000.

ANTONOVICH, D. Cutaneous leishmaniasis: a diagnostic challenge. Journal of the American Academy of Dermatology, Saint Louis, v. 50, n. 3, p. 103, 2004.

AYRES, D. C.; MARCUCCI, M. C.; GIORGIO, S. Effects of Brazilian propolis on leishmania amazonensis.
Memórias do Instituto Oswaldo Cruz, Rio de Janeiro, v. 102, n. 2, p. 215-220, 2007.

BAILEY, M. S.; LOCKWOOD, D. N. Cutaneous leishmaniasis. Clinics in Dermatology, Philadelphia, v. 25. n. 2, p. 203-211, 2007.

BANKOVA, V. S.; CASTRO, S. L.; MARCUCCI, M. C. Propolis: recent advances in research on chemistry and plant origin. Apidologie, Versailles, v. 31, n. 1, p. 3-15, 2000.

BANSKOTA, A. H.; TEZUKA, Y.; MIDORIKAWA, K.; 
MATSUSHIGE, K.; KADOTA, S. Two novel cytotoxic benzofuran derivatives from Brazilian propolis. Journal of Natural Products, Cincinnati, v. 63, n. 9, p. 12771279, 2000.

BANSKOTA, A. H.; TEZUKA, Y.; PRASAIN, J. K.; MATSUSHIGE, K.; SAIKI, J.; KADOTA, S. Chemical constituents of Brazilian propolis and their cytotoxic activities. Journal of Natural Products, Cincinnati, v. 61, n. 7, p. 896-900, 1998.

BARRAL, A.; BARRAL-NETTO, M.; YONG, E. C.; BROWNELL, C. E.; TWARDZIK, D. R.; REED, S. G. As a virulence mechanism for $\beta$ Transforming growth factor Leishmania braziliensis. The Proceedings of the National Academy of Sciences USA, Washington, v. 90, p. 3442-3446, 1993.

BARROS, M. P.; SOUSA, J. P. B.; BASTOS, J. K.; ANDRADE, S. F. Effect of Brazilian green propolis on experimental gastric ulcers in rats. Journal of Ethnopharmacology, Lausanne, v. 110, n. 3, p. 567-571, 2007.

BELKAID, Y.; PICCIRILLO, C. A.; MENDEZ , S.; SHEVACH, E. M.; SACKS, D. L. CD4+CD25+ regulatory $\mathrm{T}$ cells control leishmania major persistence and immunity. Nature, London, v. 420, n. 6915, p. 502507, 2002.

CAMPOS, R. O.; PAULINO, N.; SILVA, C. H.; SCREMIN, A.; CALIXTO, J. B. Antihiperalgesic effect of an ethanolic extract of propolis on mice and rats. Journal of Pharmacology and Pharmacology, London, v. 50, n. 10, 1187-1193, 1998.

CASTELLANO, V. M.; RODRÍGUEZ-PERALTO, J. L.; ALONSO, S.; GÓMEZ-DE LA FUENTE, E.; IBARROLA, C. Dermatofibroma parasitized by Leishmania in HIV infection: a new morphologic expression of dermal Kala Azar in an immunodepressed patient. Journal of Cutaneous Pathology, Copenhagen, v. 26, n. 10, p. 516-519, 1999.

CASTRO, E. A.; THOMAZ-SOCCOL, V.; AUGUR, C.; LUZ, E. Leishmania (Viannia) braziliensis: epidemiology of canine cutaneous leishmaniasis in the State of Parana (Brazil). Experimental Parasitology, San Diego, v. 117, n. 1, p. 13-21, 2007.

DA SILVA FILHO, A. A.; ALBUQUERQUE, S.; SILVA, M. L. A.; EBERLIN, M. N.; TOMAZELA, D. M.; BASTOS, J. K. Tetrahydrofuran lignans from Nectandra megapotamica with trypanocidal activity. Journal of Natural Products, Cincinnati, v. 67, n. 1, p. 42-45, 2004a.

DA SILVA FILHO, A. A.; BUENO, P. C. P.; GREGÓRIO, L. E.; SILVA, M. L. A.; ALBUQUERQUE, S.; BASTOS, J. K. In vitro trypanocidal activity evaluation of crude extract and isolated compounds from Baccharis dracunculifolia D. C. (Asteraceae). Journal of Pharmacology and Pharmacology, London, v. 56, n. 9, p. 1195-1199, 2004b.

DE ALMEIDA, M. C.; CARDOSO, S. A.; BARRALNETTO, M. Leishmania (Leishmania) chagasi infection alters the expression of cell adhesion and costimulatory molecules on human monocyte and macrophage. International Journal of Parasitology, Oxford, v. 33, n. 2, p. 153-162, 2003.

DURAN, G.; DURAN, N.; CULHA, G.; OZCAN, B.; OZTAS, H.; OZER B. In vitro antileishmanial activity of Adana propolis samples on Leishmania tropica: a preliminary study. Parasitology Research, Berlin, v. 102, n. 6, p. 1217-1225, 2008.

EL-ON, J.; HAMBURGER, A. D. Topical treatment of New and Old World cutaneous leishmaniasis in experimental animals. Royal Society of Tropical Medicine and Hygiene, London, v. 81, n. 5, p. 734-737, 1987.

GENARO, O. Leishmaniose tegumentar americana. In: NEVES, D. P.; MELO, A. L.; LINARDI, P. M, VITOR, R. W. A. (Ed.). Parasitologia humana. 11. ed. São Paulo: Atheneu, 2005. p. 41-83.

GRIMALDI, G. J. R.; TESH, R. B.; MCMAHONPRATT, D. A review of the geographic distribution and epidemiology of leishmaniasis in the New World. American Journal of Tropical Medicine and Hygiene, Mclean, v. 41, n. 6, p. 687-725, 1989.

HALL, B. F.; JOINER, K. A. Strategies of obligate intracellular parasites for evading host defences. Immunology Today, Cambridge, v. 12, n. 3, p. 22-27, 1991.

KAYSER, O.; KIDERLEN, A. F.; CROFT, S. L. Natural products as antiparasitic drugs. Parasitology Research, Berlin, v. 90, supp. 2, p. 55-62, 2003.

KUJUMGIEV, A.; TSVETKOVA, I.; SERKEDJIEVA, Y.; BANKOVA, V.; CHRISTOV, R.; POPOV, S. Antibacterial, antifungal and antiviral activity of propolis from different geographic origin. Journal of Ethnopharmacology, Lausanne, v. 64, n. 3, p. 235-240, 1999.

KUMAZAWA, S.; YONEDA, M.; SHIBATA, I.; KANAEDA, J.; HAMASAKA, T.; NAKAYAMA, T. Direct evidence for the plant origin of Brazilian propolis by the observation of honeybee behavior and phytochemical analysis. Chemical and Pharmaceutical Bulletin, Tokyo, v. 51, n. 6, p. 740-742, 2003.

LEITÃO, D. P. S.; DA SILVA FILHO, A. A.; POLIZELLO, A. C. M.; BASTOS, J. K.; SPADARO, 
A. C. C. Comparative evaluation of in vitro effects of Brazilian green propolis and baccharis dracunculifolia extracts on cariogenic factors of Streptococcus mutans. Chemical and Pharmaceutical Bulletin, Tokyo, v. 27, n. 11, p. 1834-1839, 2004.

LIEW, F. Y.; MILlOTT, S.; PARKINSON, C.; PALMER, R. M.; MONCADA, S. Macrophage killing of Leishmania parasite in vivo is mediated by nitric oxide from L-arginine. The Journal of Immunology, New York, v. 144, n. 12, p. 4794-4797, 1990.

LIMA, R. O. A. Mecanismos de ação da própolis na modulação de danos quimicamente induzidos no DNA. 2007. Tese (Doutorado em Patologia) - Universidade Estadual Paulista "Júlio de Mesquita Filho", Botucatu.

LUMSDEN, W. H. Letter: Biochemical taxonomy of Leishmania. Royal Society of Tropical Medicine and Hygiene, London, v. 68, n. 1, p. 74-75, 1974.

MACHADO, G. M.; LEON, L. L.; DE CASTRO, S. L. Activity of Brazilian and Bulgarian propolis against different species of leishmania. Memórias do Instituto Oswaldo Cruz, Rio de Janeiro, v. 102, n. 1, p. 73-77, 2007.

MARCUCCI, M. C. Propolis: chemical composition, biological properties and therapeutic activity. Apidologie, Versailles, v. 26, n. 2, p. 83-99, 1995.

MARCUCCI，M. C.; BANKOVA, V. S. Chemical composition, plant origin and biological activity of Brazilian propolis. Current Topics in Phytochemistry, Índia, v. 2, p. 115-123, 1999.

MARCUCCI, M. C.; DE CAMARGO, F. A.; LOPES, C. M. A. Identification of amino acidsin Brazilian propolis. Zeitschrift für Naturforschung, Tubingen, v. 51, n. 1/2, p. 11-14, 1996.

MARCUCCI, M. C.; FERRERES, F.; GARCIAVIQUERA, C.; BANKOVA, V. S.; DE CASTRO, S. L.; DANTAS, A.P.; VALENTE, P.H.; PAULINO, N. Phenolic compounds from Brazilian propolis with pharmacological activities. Journal of Ethnopharmacology, Lausanne, v. 74, n. 2, p. 105-112, 2001.

MATTNER, F.; MAGRAM, J.; FERRANTE, J.; LAUNOIS, P.; DI PADOVA, K.; BEHIN, R.; GATELY, M. K.; LOUIS, J. A.; ALBER, G. Genetically resistant mice lacking interleukin-12 are susceptible to infection with Leishmania major and mount a polarized Th2 cell response. European Journal of Immunology, Weinheim, v. 26, n. 7, p. 1553-1559, 1996.

MINISTÉRIO DA SAÚDE - Manual de controle da Leishmaniose Tegumentar Americana. Brasília/DF 2000. MISSIMA, F.; DA SILVA FILHO, A. A.; NUNES, G.
A.; BUENO, P. C. P.; SOUSA, J. P. B.; BASTOS, J. K.; SFORCIN, J. M. Effect of Baccharis dracunculifolia D.C. (Asteraceae) extracts and its isolated compounds on macrophage activation. Journal of Pharmacology and Pharmacology, London, v. 59, n. 3, p. 463-468, 2007.

MODABBER, F. Leishmaniasis. In: TROPICAL DISEASES RESEARCH PROGRESS 1991-1992. Geneve: World Health Organization, 1993. p. 77-87.

MURAD, J. M.; CALVI, S. A.; SOARES, A. M. V. C.; BANKOVA, V.; SFORCIN, J. M. Effects of propolis from Brazil and Bulgaria on fungicidal activity of macrophages against Paracoccidioides brasiliensis. Journal of Ethnopharmacology, Lausanne, v. 79, n. 3, p. 331-34, 2002.

NETO, A. G.; DA SILVA FILHO, A. A.; COSTA, J. M. L. C.; VINHOLIS, A. H. C.; SOUZA, G. H. B.; CUNHA, W. R.; SILVA, M. L. A.; ALBUQUERQUE, S.; BASTOS, J. K. Evaluation of the trypanocidal and antileishmanial in vitro activity of the crude hydroalcoholic extract of Pfaffia glomerata (Amaranthaceae) roots. Phytomedicine, Jena, v. 11, p. 662-665, 2004.

NEVES, D. P. Parasitologia humana. 10. ed. Rio de Janeiro: Atheneu, 2000.

NOBEN-TRAUTH, N.; KROPF, P.; MÜLLER, I. Susceptibility to Leishmania major infection in interleukin-4-deficient mice. Science, Washington, v. 271, n. 5251, p. 987-990, 1996.

ORSI, R. O.; BANKO, V.A. V.; SFORCIN, J. M. Effects of Brazilian and Bulgarian propolis on bactericidal activity of macrophages against Salmonella Typhimurium. International. Immunopharmacology, New York, v. 5, n. 2, p. 359-368, 2005.

ORSI, R. O.; FUNARI, S. R. C.; SOARES, A. M. V. C.; CALVI, S. A.; OLIVEIRA,S. L.; SFORCIN, J. M.; BANKOVA, V. Immunomodulatory action of propolis on macrophage activation. The Journal of Venomous Animals and Toxins, Botucatu, v. 6, n. 2, 205-219, 2000.

PADIGEL, U. M.; ALEXANDER, J.; FARRELL, J. P. The role of interleukin-10 in susceptibility of BALB/c mice to infection with Leishmania mexicana and Leishmania amazonensis. The Journal of Immunology, New York, v. 171, n. 7, p. 3705-3710, 2003.

PADIGEL, U. M.; FARRELL, J. P. CD40-CD40 ligand costimulation is not required for initiation and maintenance of a Th1-type response to Leishmania major infection. Infection and Immunity, Washington, v. 71, n. 3, p. 1389-95, 2003.

PADIGEL, U. M.; KIM, N.; CHOI, Y.; FARRELL, J. P. TRANCE-RANK costimulation is required for IL-12 production and the initiation of a Th1-type response to 
Leishmania major infection in CD40L-deficient mice. The Journal of Immunology, New York, v. 171, n. 10, p. 5437-5441, 2003.

PEREIRA, B. A. S.; ALVES, C. R. Immunological characteristics of experimental murine infection with Leishmania (Leishmania) amazonensis. Veterinary Parasitology, Amsterdam, v. 158, n. 4, p. 239-255, 2008.

PONTIN, K.; DA SILVA FILHO, A. A.; SANTOS, F. F.; ANDRADE E SILVA, M. L.; CUNHA, W. R.; NANAYAKKARA, N. P. D.; BASTO, J. K.; ALBUQUERQUE, S. In vitro and in vivo antileishmanial activities of a Brazilian green propolis extract. Parasitology Research, Berlin, v. 103, n. 3, p. 487-492, 2008.

REED, S. G.; SCOTT, P. A. T-cell and cytokine responses in leishmaniosis. Current Opinion in Immunology, London, v. 5, n. 4, p. 524-531, 1993.

REIS, A. B.; TEIXEIRA-CARVALHO, A.; GIUNCHETTI, R. C.; GUERRA, L. L.; CARVALHO, M. G.; MAYRINK, W.; GENARO, O.; CORREAAOLIVEIRA, R.; MARTINS-FILHO, O. A. Phenotypic features of circulating leucocytes as immunological markers for clinical status and bone marrow parasite density in dogs naturally infected by Leishmania chagasi. Clinical and Expimental Immunology, Oxford, v. 146, n. 2, p. 303-311, 2006.

REIS, C. M. F.; CARVALHO, J. C. T.; CAPUTO, L. R. G.; PATRÍCIO, K. C. M.; BARBOSA, M. V. J.; CHIEFF, A. L.; BASTOS, J. K. Atividade antiinflamatória, antiúlcera gástrica e toxicidade subcrônica do extrato etanólico de própolis. Brazilian journal of Pharmacognosy, Curitiba, v. 10, n. 10, p. 43-52, 2000.

RIOUX, J. A.; LANOTTE, G.; SERRES, E.; PRATLONG, F.; BASTIEN, P.; PERIERES, J. Taxonomy of Leishmania. Use of isoenzymes. Suggestions for a new classification. Annales de Parasitologie Humaine et Comparée, Paris, v. 65, n. 3, p. 111-125, 1990.

RITTER, U.; MATTNER, J.; ROCHA, J. S.; BOGDAN, C.; KÖRNER, H. The control of Leishmania (Leishmania) major by TNF in vivo is dependent on the parasite strain. Microbes and Infection, Paris, v. 6, n. 6, p. 559-565, 2004a.

RITTER, U.; MEISSNER, A.; SCHEIDIG, C.; KÖRNER, H. CD8 alpha- and Langerin-negative dendritic cells, but not Langerhans cells, act as principal antigen-presenting cells in leishmaniasis. European Journal of Immunology, Weinheim, v. 34, n. 6, p. 1542-1550, 2004b.

ROBERTS, M. T. M. Current understandings on the immunology of leishmaniasis and recent developments in prevention and treatment. British Medical Bulletin,
Edinburgh, v. 17, n. 75/76, p. 115-130, 2006.

SAHINLER, N.; KAFTANOGLU, O. Natural product propolis: chemical composition. Natural Products Research, Milton Park, v. 19, n. 2, p. 183-188, 2005.

SALOMÃO, K.; DANTAS, A. P.; BORBA, C. M.; CAMPOS, L. C.; MACHADO, D. G.; AQUINO NETO, F. R.; DE CASTRO, S. L. Chemical composition and microbicidal activity of extracts from Brazilian and Bulgarian propolis. Letters in Appiedl Microbiology, Oxford, v. 38, n. 2, p. 87-92, 2004.

SALOMÃO, K.; PEREIRA, P. R.; CAMPOS, L. C.; BORBA, C. M.; CABELLO, P. H.; MARCUCCI, M. C.; DE CASTRO, S. L. Brazilian propolis: correlation between chemical composition and antimicrobial activity. Evidence-based Complementary and Alternative Medicine, Oxford, v. 5, n. 3, p. 317-324, 2008.

SCHELLER, S.; GAZDA, G.; KROL, W.; CZUBA, Z.; ZAJUSZ, A.; GABRYS, J.; SHANI, J. The ability of ethanolic extract of propolis (EEP) to protect mice against gamma irradiation. Zeitschrift für Naturforschung: Section C, Tübingen, v. 44, n. 11/12, p. 1049-1052, 1989.

SCOTT, P.; NATOVITZ, P.; COFFMAN, R. L.; PEARCE, E.; SHER, A. Immunoregulation of cutaneous leishmaniasis. $\mathrm{T}$ cell lines that transfer protective immunity or exacerbation belong to different $\mathrm{T}$ helper subsets and respond to distinct parasite antigens. The Journal of Experimental Medicine, New York, v. 168, n. 5, p. 1675-1684, 1988.

SFORCIN, J. M.; FERNANDES, A. J. R.; LOPES, C. A.; BANKOVA, V.; FUNARI, S. R. Seasonal effect on Brazilian propolis antibacterial activity. Journal of Ethnopharmacology, Lausanne, v. 73, n. 1/2, p. 243-249, 2000.

SFORCIN, J. M.; ORSI, R. O.; BANKOVA, V. Effect of propolis, some isolated compounds and its source plant on antibody production. Journal of Ethnopharmacology, Lausanne, v. 98, n. 3, p. 301-305, 2005.

SILVEIRA, T. G.; ARRAES, S. M.; BERTOLINI, D. A.; TEODORO, U.; LONARDONI, M. V.; ROBERTO, A. C.; RAMOS, M.; SOBRINHO, A. N.; ISHIKAWA, E.; SHAW, J. The laboratory diagnosis and epidemiology of cutaneous leishmaniasis in Parana State, southern Brazil. Revista da Sociedade Brasileira de Medicina Tropical, Uberaba, v. 32, n. 4, p. 413-23, 1999.

SIMÕES, L. M. C.; GREGORIO, L. E.; DA SILVA FILHO, A. A.; DE SOUZA, M. L.; AZZOLINI, A. E. C. S.; BASTOS, J. K.; LUCISANO-VALIM, Y. M. Effect of Brazilian green propolis on the production of reactive oxygen species by stimulated neutrophils. Journal of Ethnopharmacology, Lausanne, v. 94, n. 1, p. 59-65, 
2004.

SOLBACH, W.; LASKAY, T. The host response to Leishmania infection. Advances in Immunology, San Diego, v. 74, p. 275-317, 2000.

TATEFUJI, T.; IZUMI, N.; OHTA, T.; ARAI, S.; IKETA, M.; KURIMOTO, M. Isolation and identification of compounds from Brazilian própolis which enhance macrophage spreading and motility. Biological and Pharmaceutical Bulletin, Tokyo, v. 19, n. 7, p. 966-970, 1996.

TAVARES, D. C.; MAZZARON BARCELOS, G. R.; SILVA, L. F.; CHACON TONIN, C. C.; BASTOS, J. K. Propolis induced genotoxicity and antigenotoxicity in Chinese hamster ovary cells. Toxicology In Vitro, Oxford, v. 20, n. 7, p. 1154-1158, 2006.

TRIPATHI, P.; SINGH, V.; NAIK, S. Immune response to leishmania: paradox rather than paradigm. FEMS Immunology and Medical Microbiology, Amsterdam, v. 51, n. 2, p. 229-42, 2007.

VON STEBUT, E.; BELKAID, Y.; JAKOB, T.; SACKS, D. L.; UDEY, M. C. Uptake of leishmania major amastigotes results in activation and interleukin 12 release from murine skin-derived dendritic cells: implications for the initiation of anti-Leishmania immunity. The Journal of Experimental Medicine, New York, v. 188, n. 8, p. 1547-1552, 1998.

WANG ZE, REINER SL, ZHENG S, DALTON DK, LOCKSLEY RM. CD4+ effector cells default to the Th2 pathway in interferon gamma-deficient mice infected with Leishmania major. J Exp Med 1994;179:1367-71.

WORLD HEALTH ORGANIZATION. Leishmaniases. 2002. Availabre in: <http://www.who.int/ zoonoses/ diseases/leishmaniasis/en/>. Access in: 29 Sep. 2010.

ZIMMERMANN, S.; MOLL, H.; SOLBACH ,W.; LÜDER, C. G. Meeting Report IFoLeish-2008: current status and future challenges in leishmania research and leishmaniasis. Protist, Jena, v. 160, n. 2, p. 151-158, 2009.

Recebido em 3 de abril de 2010-Received on April 3, 2010

Aceito em 22 de setembro de 2010 - Accepted on September 22, 2010 